\title{
Les matériaux pour les composants proches du plasma dans les réacteurs à confinement magnétique
}

ANA ALAMO, LUCIANO GIANCARLI, JEAN JACQUINOT, GEORGES MARTIN, YVES POITEVIN, EMMANUEL RIGAL 

Les études concernant les interactions d'un plasma avec les matériaux l'entourant constituent l'un des thèmes majeurs d'Iter. Au strict niveau des composants face au plasma, des réponses fondamentales seront apportées par lter quant à leur comportement en présence d'un plasma de plusieurs centaines de secondes (tenue à l'érosion en particulier). Ces expérimentations permettront sans doute déjà de disqualifier certains matériaux pour une utilisation en réacteur. La mise en place d'un programme de R\&D volontaire sur les matériaux susceptibles $d^{\prime}$ être candidats dans un réacteur (tungstène, carbure de silicium, voire des matériaux carbonés si lter ne les condamne pas) est capitale pour le développement de la fusion. Par rapport à lter, le réacteur ajoutera des contraintes supplémentaires liées à des exigences de production d'énergie, comme par exemple un fonctionnement continu et des exigences de rentabilité maximale (fonctionnement à des températures $>500^{\circ} \mathrm{C}$ ). Les performances $\mathrm{d}^{\prime}$ lter en termes de durée $d^{\prime}$ 'impulsion ont permis I'utilisation d'un acier classique comme matériau de structure (acier austénitique de type 316), matériau incompatible en environnement réacteur de fusion à cause de son gonflement trop important sous irradiation. La parade existe avec l'utilisation des aciers martensitiques à $9 \%$ $\mathrm{Cr}$, largement étudiés dans le cadre des réacteurs à neutrons rapides et qui ne gonflent pas jusqu'à des doses de $150 \mathrm{dpa}$, niveau compatible avec la conception d'un réacteur de fusion. La composition de ces aciers a été optimisée dans le cadre des activités «fusion » afin de limiter leur activation ou plus exactement $\mathrm{d}^{\prime}$ accélérer leur désactivation. Mais le neutron de fusion possède une caractéristique bien particulière qui est son énergie de $14 \mathrm{MeV}$. Cette énergie donne accès à des réactions de transmutation à l'origine de production d'hélium et

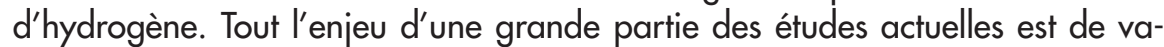
lider le comportement de ces matériaux dans un environnement associant une dose importante et une production de gaz. Ceci passe par la mise en place d'un programme de modélisation (et des moyens expérimentaux dédiés) conséquent, devant permettre l'optimisation de futurs programmes de validation technologique dans une source intense de neutrons de $14 \mathrm{MeV}$. Une telle source est l'élément central du projet IFMIF (International Fusion Materials Irradiation Facility) réalisé sous I'auspice de l'IEA (International Energy Agency) dont les partenaires majeurs sont l'Europe et le Japon.

Ces matériaux constitueront le squelette de composants très complexes, multifonctions comme les couvertures. Ces dernières devront assurer dans un réacteur trois fonctions principales : la production du tritium, l'extraction de la chaleur, et une protection neutronique des parties extérieures à la chambre à vide. Des exigences de rendement élevé et de fiabilité sont bien évidement des contraintes supplémentaires à prendre en compte. La mise au point de procédés de fabrication des tels composants, satisfaisant à toutes les contraintes imposées à une couverture tritigène est une thématique importante pour la crédibilité de l'énergie de fusion. 


\section{Problématique et solutions potentielles pour les composants proches du plasma}

Par rapport à lter, les principaux problèmes qui se posent dans les composants proches du plasma de Demo et des futurs réacteurs à fusion sont essentiellement :

- l'effet des irradiations neutroniques sur le comportement des matériaux qui les composent, en particulier, l'effet de la production d'hélium ;

- le niveau élevé des températures de fonctionnement induites par la recherche de rendements thermodynamiques attractifs;

- la limitation de l'activation des matériaux qu'il faut gérer au-delà de leur passage en réacteur.

Pour illustrer les progrès à accomplir sur ces trois aspects, on peut retenir notamment que :

- les matériaux de structure des réacteurs à fission reçoivent des dommages neutroniques de l'ordre de $1 \mathrm{dpa}$, alors dans un réacteur à fusion, l'objectif est de pouvoir atteindre environ $150 \mathrm{dpa}$ au niveau de la première paroi des couvertures;

- certains composants proches du plasma devront mettre en œuvre des fluides réfrigérants dont la température maximale en sortie pourrait se situer dans une fourchette de $500^{\circ} \mathrm{C}$ à plus de $1000^{\circ} \mathrm{C}$.

Il est clair que les problèmes posés et les solutions proposées dépendent fortement du type et des fonctionnalités des composants considérés. En particulier, les principales structures proches du plasma recouvrent :

- les couvertures tritigènes, incluant la première paroi, qui ont trois fonctions principales : la production du tritium, l'extraction de la chaleur, et une contribution à la protection neutronique des parties extérieures à la chambre à vide. Le caloporteur et les circuits de refroidissement associés à la couverture détermineront le rendement électrique de l'installation l'objectif est une température de sortie la plus élevée possible, à partir de $500^{\circ} \mathrm{C}$ jusqu'à plus de $1000^{\circ} \mathrm{C}$ dans une machine industrielle construite dans la seconde partie du siècle). Les couvertures sont soumises à plusieurs contraintes supplémentaires, et parfois contradictoires, telles que l'obligation d'assurer l'autosuffisance en tritium et une bonne tenue mécanique sous irradiation accompagnée d'un haut niveau d'étanchéité ; 
- les composants à haut flux, et en particulier le divertor, cendrier de la réaction de fusion, soumis à des flux surfaciques intenses (de 5 à $15 \mathrm{MW} / \mathrm{m}^{2}$ ), en contact avec le plasma, devront présenter une bonne conductibilité thermique et en plus, ce qui n'était pas nécessaire dans lter, une bonne tenue à l'irradiation ;

- les protections neutroniques, installées à l'arrière des couvertures et du divertor dans la chambre à vide, qui ont le double objectif de réduire à des niveaux acceptables le dépôt de chaleur dans les bobines supraconductrices et de limiter la fluence neutronique au niveau des connections et des attachement des couvertures afin de rendre possible leur remplacement périodique.

La conception des structures proches du plasma est nécessaire pour définir les types de matériaux envisageables et leurs conditions de fonctionnement, et donc détermine le niveau et la quantité de R\&D nécessaires pour arriver à la conception d'un réacteur électrogène. Deux composants seront particulièrement difficiles à réaliser : les couvertures tritigènes et le divertor.

\subsection{Les couvertures tritigènes}

Il faut rappeler que, devant la quantité de tritium nécessaire pour alimenter un réacteur de fusion thermonucléaire (un réacteur de $900 \mathrm{MWe}$ consomme plus de $300 \mathrm{~g}$ de $\mathrm{T}$ par jour), il est nécessaire d'utiliser les neutrons issus du plasma pour transmuter le lithium in situ, dans des couvertures tritigènes, et assurer ainsi l'autosuffisance du réacteur.

En tenant compte des quantités de tritium limitées disponibles aujourd'hui, cette condition devra être déjà respectée dans Demo, l'étape suivant Iter. Cette contrainte implique la mise en œuvre d'un vaste programme de R\&D, soutenu par une importante réflexion en termes de conception et $d^{\prime}$ analyse.

Chaque réaction de fusion consomme un noyau de tritium et produit un neutron de $14 \mathrm{MeV}$ qui peut être utilisé pour produire un noyau de tritium par réaction nucléaire avec un noyau de lithium- 6 nécessairement présent dans la couverture. Comme chaque neutron peut produire un seul noyau de tritium, on voit facilement que, si on tient compte des neutrons absorbés par d'autres noyaux dans les matériaux de la couverture (" réactions parasites 》 et des fuites à travers les zones autours du plasma où il n'y a pas de couverture, telles que la région du divertor), l'autosuffisance en tritium ne peut être atteinte qu'avec l'utilisation de matériaux multiplicateurs de neutrons, via les réactions du type (n, $2 n)$. Les seuls matériaux multiplicateurs suffisamment performants qu'on peut envisager sont le plomb et le béryllium. En principe, il y a une troisième possibilité 
qui consisterait en l'utilisation, sous certaines conditions assez contraignantes, du ${ }^{7} \mathrm{Li}$ disponible dans le lithium naturel à travers une réaction $(n, t)$.

La conception des couvertures tritigènes doit donc optimiser la combinaison entre plusieurs matériaux nécessaires, à savoir :

- un matériau producteur de tritium, donc contenant du lithium (par exemple, le lithium liquide, l'eutectique $\mathrm{Li} \mathrm{Pb}$, des céramiques lithiées, ou des sels fondus incluant du lithium);

- un multiplicateur de neutron (le plomb ou le béryllium);

- un matériau de structure (l'acier, les alliages de vanadium, le $\mathrm{SiC} / \mathrm{SiC}$ );

- un caloporteur (I'eau pressurisée, l'hélium ou un métal liquide). Toutes les combinaisons ne sont cependant pas possibles et la recherche d'une solution doit prendre en compte des critères de compatibilités, les performances demandées, les contraintes de sûreté, la minimisation des déchets radioactifs, et, bien sûr, les coûts.

En Europe, un programme de développement pour les couvertures tritigènes, auquel le CEA a activement participé depuis le début, est en cours depuis les années 1980. Les études menées dans le cadre de ce programme ont permis de sélectionner deux concepts de couverture (et donc deux quadruplets matériaux : tritigène/multiplicateur/structure/réfrigérant) pour Demo, qui seront testés dans Iter. Les deux concepts sont les suivants :

- la couverture HCLL (Helium-Cooled Lithium-Lead), qui utilise un eutectique du lithium-plomb (Pb-17Li) comme multiplicateur de neutrons et producteur de tritium, des structures en acier ferritique-martensitique à basse activation développé spécialement pour la fusion, appelé Eurofer, et enfin I'hélium comme caloporteur; I'hélium est à une pression de $8 \mathrm{MPa}$ et les températures $d^{\prime}$ entrée et sortie sont respectivement de $300{ }^{\circ} \mathrm{C}$ et $500{ }^{\circ} \mathrm{C}$. L'eutectique $\mathrm{Pb}-17 \mathrm{Li}$, dont la température de fusion est de $235^{\circ} \mathrm{C}$, est présent sous forme liquide et il circule à quelques $\mathrm{mm} / \mathrm{s}$ pour permettre l'extraction du tritium à l'extérieur de la chambre à vide. Les analyses neutroniques ont montré qu'ainsi l'autosuffisance en tritium est assurée. Plusieurs points critiques ont cependant été identifiés et sont en cours d'évaluation au sein d'un programme de R\&D, coordonné au niveau européen sous responsabilité du CEA, et mené dans plusieurs Associations. Ils concernent les aspects "procédés de fabrication » des structures, le contrôle et la gestion du tritium (ce qui a nécessité le développement de barrières de perméation, incluant la tenue sous irradiation et l'étude de procédés d'extraction du tritium du Li Pb et de l'hélium), la compatibilité 
de l'acier des structures avec le $\mathrm{Li}$ Pb (et le développement éventuel de barrières de corrosion), le développement des composants des circuits hélium, et la maîtrise des phénomènes MHD;

- la couverture HCPB (Helium-Cooled Pebble Bed) utilisant des céramiques lithiées sous forme de lits de billes (Li4SiO4, Li2TiO3) comme producteur de tritium, le béryllium sous forme de lits de billes comme multiplicateur de neutron, l'Eurofer comme matériau de structure et l'hélium comme caloporteur dans les mêmes conditions que la couverture HCLL. L'extraction du tritium est obtenue grâce à un balayage d'hélium à basse pression $(0,1 \mathrm{MPa})$ à travers les lits de billes. Les céramiques doivent avoir une température comprise entre $600{ }^{\circ} \mathrm{C}$ et $900{ }^{\circ} \mathrm{C}$. Pour ce concept aussi, l'autosuffisance en tritium a été démontrée avec des calculs utilisant des modèles 3D. Le programme de R\&D associé est coordonné au niveau européen sous la responsabilité du centre de recherche allemand de Karlsruhe. Outre les aspects " procédés de fabrication " des structures et circuits hélium, communs avec le concept HCLL, les points critiques adressés concernent surtout le comportement des lits des billes de céramiques et de béryllium sous contraintes thermiques et sous irradiations, leur compatibilité avec le matériau de structure, le relâchement du tritium produits dans les céramiques et dans le béryllium, la gestion du problème de l'inventaire en tritium, surtout dans le béryllium.

Ces deux concepts de couvertures seront testés dans le réacteur Iter sous la forme de maquettes dédiées et optimisées pour reproduire au plus près les conditions opérationnelles attendues dans le réacteur Demo.

Bien sûr le développement du matériau de structure Eurofer, commun aux deux concepts, a un poids significatif dans le programme de R\&D pour les couvertures et il sera détaillé dans le chapitre suivant.

Dans le cadre d'une étude européenne d'un réacteur électrogène (après Demo) qui a été menée entre 2001 et 2003 (PPCS, Power Plant Conceptual Studies), des concepts de couvertures plus avancés ont été identifiés et évalués. Ces études visaient à identifier les concepts de composants internes d'un réacteur commercial en tenant compte des contraintes économiques inhérentes à la production électrique, de la sûreté et de la gestion des déchets radioactifs. Cette étude a pris en compte, pour la première fois en Europe, tous les aspects d'intégration du réacteur, à savoir, les compromis nécessaires entre l'optimisation du fonctionnement de chaque composant et les exigences dictées par les aspects globaux tels que la maintenance, la télémanipulation, la sûreté, l'accès dans la chambre à vide, et, bien sûr, le fonctionnement du plasma. L'objectif était de définir, en partant des concepts envisagés, les principaux axes de R\&D qui devront être privilégiés dans les prochaines décennies. 
Quatre modèles de réacteur ont été retenus dans cette étude. Les deux premiers se réfèrent aux deux types de couverture envisagés pour Demo et décrits ci-dessus, qui peuvent assurer un rendement thermique global de l'ordre de $40 \%$ (à noter que le modèle utilisant la couverture $\mathrm{Li} \mathrm{Pb}$ a été initialement étudié dans une version refroidie à l'eau). Les deux autres modèles envisagent l'utilisation de couvertures plus innovantes : la couverture « Dual Coolant» (DC) et la couverture Li Pb autorefroidie (Self-Cooled Lithium Lead, SCLL).

La couverture « Dual Coolant » (DC) utilise comme matériau de structure l'Eurofer. Le concept consiste essentiellement en une boîte, dont les structures sont refroidies par de l'hélium, qui contient du lithium-plomb circulant à haute vitesse, permettant ainsi l'extraction de la chaleur déposée dans son propre volume (proche $d^{\prime}$ un concept dit « autorefroidi »). Afin de contourner les problèmes de MHD, le lithium-plomb est isolé électriquement des parois en acier par des inserts en $\mathrm{SiC} / \mathrm{SiC}$ dont le développement nécessite un effort important. Ces inserts agissent aussi comme isolants thermiques, ce qui permet d'avoir une température de sortie du $\mathrm{Pb}$-17Li largement supérieure à la température maximale acceptable par l'acier. La température de sortie du $\mathrm{Li} \mathrm{Pb}$ peut ainsi atteindre plus de $700{ }^{\circ} \mathrm{C}$ et le rendement peut atteindre environ $45 \%$.

La couverture SCLL est un concept encore plus avancé, qui extrapole au maximum les connaissances physiques et technologiques actuelles; en effet, ce modèle exploite les caractéristiques favorables de la fusion D-T en termes de sûreté et maintenance et les associe au fonctionnement à très haute température conduisant à des rendements thermiques très élevés. Il est basé sur une couverture " autorefroidie » au lithium-plomb avec des structures en $\mathrm{SiC} / \mathrm{SiC}$. En principe, il peut être associé à un divertor autorefroidi au lithium-plomb avec des structures en $\mathrm{SiC} / \mathrm{SiC}$ et des tuiles de protection en tungstène, permettant d'espérer atteindre des flux surfaciques de l'ordre de $5 \mathrm{MW} / \mathrm{m}^{2}$. L'inconnue principale provient de l'utilisation du $\mathrm{SiC} / \mathrm{SiC}$ comme matériau de structure, notamment en termes de conductivité thermique, de résistance mécanique et de tenue sous irradiation. Avec des températures de sortie du caloporteur voisinant les $1100{ }^{\circ} \mathrm{C}$, le rendement pour ce modèle de réacteur pourrait atteindre environ $58 \%$.

\subsection{Le divertor}

Le divertor est un composant déjà soumis à des contraintes extrêmes dans le cadre d'Iter, et ses matériaux constitutifs sont en limite de capacité en termes de comportement sous irradiation. L'étude d'un concept potentiel pour Demo et audelà $n^{\prime}$ a commencé que très récemment, tout au moins en Europe. Pour résister à un tel niveau d'irradiation, seul le tungstène est acceptable comme matériau de protection; pour la partie structure, les matériaux de structures déjà utilisé dans les couvertures (Eurofer, $\mathrm{SiC} / \mathrm{SiC}$ ) ou le tungstène doivent être envisagés. 
Trois concepts, tous présentant des points critiques majeurs, ont été développés en Europe dans le cadre du PPCS et peuvent être cités comme exemples:

- le concept utilisant des tubes en Eurofer refroidis à l'eau pressurisée, entourés par des monoblocs en tungstène qui ont uniquement une fonction de protection. Ce concept est théoriquement capable de résister à des flux surfaciques d'environ $7 \mathrm{MW} / \mathrm{m}^{2}$, qui pourraient être augmentés jusqu'à $15 \mathrm{MW} / \mathrm{m}^{2}$ si on ajoute une barrière thermique en graphite entre l'acier et les monoblocs ainsi qu'une épaisseur de graphite souple (PAPYEX ${ }^{(R)}$ ) afin d'absorber l'expansion différentielle. Le comportement sous irradiation de ces matériaux reste à évaluer ;

- le concept utilisant le tungstène (ou, éventuellement, une alliage spécifique à base de tungstène encore à identifier) directement comme matériaux de structures et I'hélium comme caloporteur. Ce concept est capable de résister à des flux surfaciques de l'ordre de $10 \mathrm{MW} / \mathrm{m}^{2}$, en prenant en compte les propriétés du tungstène avant irradiation. Le fenêtre d'utilisation du tungstène est $600^{\circ} \mathrm{C}$ à $1000^{\circ} \mathrm{C}$, ce qui demande une température de sortie de l'hélium à plus de $700^{\circ} \mathrm{C}$;

- le concept autorefroidi au $\mathrm{Li} \mathrm{Pb}$, utilisant des structures en $\mathrm{SiC} / \mathrm{SiC}$ avec des tuiles de protection en tungstène. Comme dans les couvertures, la température de sortie du $\mathrm{Li} \mathrm{Pb}$ peut être proche de $1000^{\circ} \mathrm{C}$. Le comportement sous irradiation du $\mathrm{SiC} / \mathrm{SiC}$ est, bien sûr, le point critique majeur. À cause de la relativement faible conductivité thermique du $\mathrm{SiC} / \mathrm{SiC}$, ce concept est capable d'accepter un flux surfacique d'environ $5 \mathrm{MW} / \mathrm{m}^{2}$.

Seule la R\&D sur les matériaux utilisés qui devra être lancée dans les années à venir pourra répondre aux questions concernant la faisabilité de ces concepts et leur possible utilisation dans le divertor d'un réacteur.

\section{2 | Les matériaux de structure}

En général, les matériaux de structure pour un réacteur de fusion devront répondre aux exigences suivantes :

- des propriétés adéquates pour supporter les chargements mécaniques, thermiques et électromagnétiques;

- une dégradation contrôlable de propriétés et compatible avec la durée de vie envisagée du composant, cette dégradation étant due à l'irradiation 
(déplacements atomiques, production de l'hélium et hydrogène, transmutation) et dans le cas de composants face au plasma, au fort chargement thermique et le bombardement par de particules chargées;

- une faible activation après exposition au flux neutronique;

- une bonne compatibilité chimique avec le caloporteur ;

- une faible absorption du tritium, ou bien, il faudra disposer de méthodes efficaces de " détritiation ».

Les principales différences avec les exigences pour les matériaux utilisés dans les réacteurs de fission résultent du fait de chargements électromagnétiques liés, en particulier, aux disruptions du plasma, au flux de particules chargées et à l'énergie relativement élevée de neutrons ( $14 \mathrm{MeV}$ ).

Pour répondre aux critères de faible activation, trois types de matériaux peuvent en général être envisagés : les aciers ferritiques/martensitiques, les alliages base vanadium et les composites céramiques à base de carbure de silicium (SiC).

Le programme de développement technologique des matériaux pour la fusion thermonucléaire comporte plusieurs étapes, qui sont liées aux impératifs de performances accrues requises des différentes machines. Ainsi, la stratégie adoptée en Europe comprend des objectifs à échéances graduées auxquelles sont associés différents types de matériaux à qualifier, à savoir :

- "Objectif court terme » : il porte sur le développement d'un matériau de structure permettant de supporter un dommage neutronique de 70-80 dpa et une température maximale de fonctionnement de $550^{\circ} \mathrm{C}$ (conditions Demo). Ceci a donné lieu au développement d'aciers martensitiques au chrome dits "à activation réduite » ou « faible activation 》, qui seront utilisés pour la fabrication des modules « test » dans Iter;

- "Objectif à moyen terme »: il vise le développement de matériaux avancés à base fer, les aciers renforcés par une dispersion d'oxydes couramment appelés «ODS » (Oxide Dispersion Strengthening), qui peuvent accepter un niveau d'endommagement semblable (70-80 dpa) voire supérieur et fonctionner à températures plus élevées $\left(600-900^{\circ} \mathrm{C}\right)$;

- "Objectifà long terme »: il vise la qualification d'un matériau de structure à faible activation pour un réacteur prototype susceptible de fonctionner cinq ans avec un chargement neutronique de $2,5 \mathrm{MW} / \mathrm{m} 2$ supportant un dommage de $150 \mathrm{dpa}$ et des températures de fonctionnement comprises entre 500 et $1200^{\circ} \mathrm{C}$. On s'intéresse dans ce cas à la mise au point de 
composites $\mathrm{SiC}_{f} / \mathrm{SiC}$ constitués d'une matrice céramique en carbure de silicium renforcée par des fibres de même nature.

Des alliages base vanadium de type $\mathrm{V}$ - $\mathrm{Cr}$ - $\mathrm{Ti}$, sont aussi de candidats potentiels pour divers types de structures et sont étudiés notamment aux États-Unis.

Pour les applications spécifiques du divertor, les études menées en Europe portent sur les alliages base cuivre et différents métaux et alliages réfractaires tels que le tungstène et le molybdène, envisagés aussi bien pour utilisation comme protection que comme élément de la structure.

L'expérience et les données obtenues jusqu'à présent dans un environnement représentatif $d$ 'un réacteur de fusion sont très limitées. En particulier, les études concernant le comportement de matériaux sous irradiation sont effectuées dans des réacteurs de fission, qui ne permettent pas toutefois de simuler le dommage neutronique et les produits de transmutation qui seraient induits dans l'environnement $d$ 'un réacteur de fusion. La disponibilité d'une source de neutrons de $14 \mathrm{MeV}$ s'avère indispensable à la validation de matériaux de structure.

\subsection{R\&D Matériaux pour la technologie fusion}

\subsubsection{Les aciers ferritiques-martensitiques à activation réduite}

Cette classe de matériaux est actuellement la référence pour les structures susceptibles de subir un fort dommage d'irradiation en service. La conception de ceux-ci est inspirée des aciers au chrome 7-12\% Cr conventionnels qui ont fait preuve $d^{\prime}$ un très bon comportement hors et sous irradiation. En effet, dans le cadre d'études de matériaux pour les structures de cœur de réacteurs rapides, ces matériaux ont montré une grande stabilité de propriétés et une excellente résistance aux variations dimensionnelles induites par l'irradiation (gonflement, fluage $d^{\prime}$ irradiation) dans la gamme de températures d'irradiation $400-550{ }^{\circ} \mathrm{C}$.

Pour répondre aux critères de faible activation, la composition des aciers martensitiques pour la fusion a été ajustée en fonction du temps de décroissance radioactive de chaque élément constituant l'alliage. Ainsi, certains éléments à désactivation très lente, présents dans les matériaux commerciaux, tels que le molybdène, le niobium ou le nickel, ont été remplacés par d'autres comme le tungstène ou le tantale, ayant une décroissance plus rapide de leur radioactivité résiduelle après service.

La spécification d'un acier à activation réduite exige aussi un niveau très faible et bien contrôlé d'impuretés, ce qui contribue en principe à un meilleur 
comportement mécanique de l'alliage. En effet, les recherches menées dans différents laboratoires d'Europe, du Japon et des États-Unis ont confirmé l'amélioration des propriétés mécaniques par rapport aux aciers conventionnels ayant servi de base à leur développement.

D'autre part, une première expérience industrielle de fabrication d'un lingot de plusieurs tonnes répondant à la spécification d'un acier à activation réduite a eu lieu récemment en Europe. Cet acier appelé « Eurofer 97 » $9 \mathrm{Cr} 1 \mathrm{WTaV})$, constitue la référence européenne des aciers ferritiques-martensitiques à activation réduite et fait l'objet d'un vaste programme de caractérisation hors et sous irradiation. La mise en forme de ce matériau sous forme de plaques, barres ou tubes est relativement aisée et l'assemblage (par soudure TIG, faisceau d'électrons ou laser) ne pose pas de problèmes majeurs. La fabricabilité de cette classe $d^{\prime}$ acier à l'échelle industrielle est donc démontrée.

Les premiers résultats concernant les performances de l'acier Eurofer montrent une amélioration significative de ses propriétés mécaniques par rapport aux aciers martensitiques conventionnels, en particulier en ce qui concerne les valeurs de résilience et ténacité. Comme tous les matériaux métalliques, les aciers ferritiques/martensitiques sont ductiles à haute température et fragiles à basse température. La température de transition ductile-fragile (TTDF) augmente sous irradiation et cette augmentation est $d^{\prime}$ autant plus importante que la température $d^{\prime}$ irradiation diminue, en particulier pour la gamme de températures inférieures à $400^{\circ} \mathrm{C}$. Pour un fonctionnement sûr des installations, on exige que la TTDF soit inférieure à $20^{\circ} \mathrm{C}$.

La perte de ductilité et la dégradation de la ténacité associées à l'accroissement de la TTDF détermineront la durée de vie d'une structure, d'où l'importance $d$ 'étudier son évolution en conditions proches ou simulant celles envisagées lors du service. Plusieurs expériences dans des réacteurs à fission sont en cours dans la gamme $200-350^{\circ} \mathrm{C}$ afin d'étudier et quantifier les phénomènes de fragilisation et durcissement induits par l'irradiation dans ces aciers à activation réduite. La microstructure des aciers martensitiques étant très complexe, un effort important de compréhension et de modélisation est nécessaire afin d'élucider les mécanismes responsables de l'évolution du comportement mécanique.

Mis à part le dommage d'irradiation qui se traduit par de déplacements atomiques, l'autre effet attendu dans le cas d'irradiation par les neutrons de $14 \mathrm{MeV}$ est la production d'hélium et d'hydrogène générés par les réactions de transmutation. Selon les calculs prévisionnels, la fraction d'atomes d'hélium produits par rapport au nombre de déplacements atomiques serait de l'ordre de 10 appm He/dpa pour les conditions de fonctionnement de Demo. La présence de ces éléments ne fera qu'accélérer la dégradation des propriétés de matériaux, en particulier dans le domaine des températures inférieures à 
$400{ }^{\circ} \mathrm{C}$. Cependant, la production d'hélium n'atteint qu'un rapport de l'ordre de $\sim 0,1$ appm $\mathrm{He} / \mathrm{dpa}$ dans les réacteurs de fission.

La disponibilité d'une source de neutrons de $14 \mathrm{MeV}$, permettant de tester les matériaux dans un environnement représentatif $d^{\prime}$ un réacteur de fusion, s'avère indispensable à la validation et prédiction du comportement de matériaux de structure.

Du point de vue expérimental, théorique et de la modélisation, les objectifs du programme R\&D en cours sur les aciers martensitiques à activation réduite sont focalisés sur les aspects suivants :

- le comportement sous irradiation neutronique et, en particulier, les phénomènes de fragilisation à températures inférieures à $400^{\circ} \mathrm{C}$ afin de limiter l'évolution de la TTDF selon le critère d'utilisation exigé (TTDF $<20^{\circ} \mathrm{C}$ ). La température minimale de service sera déterminée par le degré de fragilisation du matériau ;

- la contribution au dommage d'irradiation due à la production d'hélium et $d$ 'hydrogène, qui peuvent précipiter sous forme de bulles de gaz, et avoir des répercussions importantes sur l'évolution de la micro/nanostructure et le comportement mécanique;

- la compatibilité avec d'autres matériaux envisagés pour les couvertures, notamment les métaux liquides $\mathrm{Pb}$ Li utilisés en tant que producteur de tritium ou multiplicateur de neutrons ou l'hélium comme caloporteur;

- le comportement en fluage thermique, qui définit la température maximale $\mathrm{d}^{\prime}$ utilisation. En général, cette dernière est $\mathrm{d}^{\prime}$ environ $550^{\circ} \mathrm{C}$. Pour Demo, le critère utilisé exige à $550{ }^{\circ} \mathrm{C}$, une résistance supérieure à $100 \mathrm{MPa}$ pendant 20000 heures et pour une dose de $70 \mathrm{dpa}$. En présence du $\mathrm{Pb}$ Li liquide, la température maximale pourrait vraisemblablement se limiter à $500^{\circ} \mathrm{C}$.

Les recherches sur ces différents aspects permettront de qualifier ce type de matériaux et de définir le domaine de températures dans lequel on pourra les utiliser pour les structures proches du plasma dans les réacteurs de fusion.

\subsubsection{Les alliages renforcés par dispersion d'oxydes (ODS)}

Les alliages à base fer renforcés par dispersion d'oxydes (ODS) ont un vaste domaine $\mathrm{d}^{\prime}$ application en raison de leur excellente résistance mécanique à des températures supérieures à $550^{\circ} \mathrm{C}$. 
Dans le cadre du programme " fusion 》, les matériaux ODS étudiés sont des aciers ferritiques-martensitiques fer-chrome de composition proche du groupe précédemment décrit, dont la matrice est renforcée par la distribution homogène de particules nanométriques d'oxydes.

Les ODS sont envisagés pour utilisation, soit comme matériau fonctionnel sous forme de placage sur une structure en Eurofer afin de la protéger d'incursions locales de températures, soit comme matériau de structure proprement dit. La référence actuelle en Europe est un ODS ayant une matrice de même composition que l'Eurofer ( $9 \mathrm{Cr} 1 \mathrm{wVTa}$ ) et une distribution de particules d'oxyde d'yttrium $\left(\mathrm{Y}_{2} \mathrm{O}_{3}\right)$, qui pourrait assurer une température maximale de fonctionnement de $700-750{ }^{\circ} \mathrm{C}$.

Pour des températures supérieures à $750^{\circ} \mathrm{C}$, le développement de nouveaux alliages ODS à plus forte teneur en chrome $(>12 \%)$ et répondant aux critères de activation réduite s'avère nécessaire.

La fabrication des ODS fait appel à des techniques très pointues de la métallurgie des poudres. En effet, ces matériaux sont élaborés par mécanosynthèse (mechanical alloying), à partir des constituants sous forme de poudres élémentaires ou pré-alliées. Dans le cas d'ODS à base fer, la mécanosynthèse est la seule méthode permettant $d$ 'introduire dans la matrice métallique la distribution fine et homogène d'oxydes qui confère au matériau la résistance mécanique à chaud optimale. Enfin, les poudres ainsi obtenues sont compactées et filées à température et pression élevées.

À l'aide de cette méthode particulière d'élaboration, des matériaux ODS ont été obtenus avec des propriétés mécaniques à haute température très encourageantes. Cependant, les performances de ce type de matériaux dépendent de la maîtrise de différents procédés intervenant dans la gamme de fabrication. Sur ce point, il faut remarquer que l'expérience existante en Europe concernant l'élaboration d'ODS est très limitée, même au niveau des laboratoires.

L'application des ODS comme matériaux de structure nécessite donc la mise en œuvre préalable d'un programme de recherche dédié à :

- la mise au point et l'optimisation des procédés de fabrication (mécanosynthèse, consolidation de poudres) afin d'obtenir des produits de bonne qualité ayant des propriétés reproductibles;

- le développement de méthodes d'assemblage en phase solide. Les procédés impliquant le passage par phase liquide sont à exclure afin de ne pas perturber la distribution homogène de particules nanométriques ; 
- la démonstration de la faisabilité à niveau industriel d'alliages ayant une composition optimisée pour les applications fusion;

- le comportement sous irradiation dans une gamme large de températures $d^{\prime}$ irradiation : la susceptibilité à la fragilisation à $\mathrm{T}<400{ }^{\circ} \mathrm{C}$, les modifications microstructurales induites par l'irradiation, I'effet de l'hélium et du rapport He appm/dpa, l'effet de l'hydrogène.

\subsubsection{Les composites céramiques $\mathrm{SiC}_{\mathrm{f}} / \mathrm{SiC}$}

Les matériaux composites céramiques sont considérés aujourd'hui comme la solution la plus plausible pour les futurs réacteurs commerciaux. Leurs propriétés à haute température sont connues pour être meilleures que celles de métaux ou $d^{\prime}$ 'alliages même réfractaires et ils présentent un intérêt majeur pour atteindre des températures supérieures à $1000^{\circ} \mathrm{C}$.

Par ailleurs, il est bien connu que ces céramiques présentent des valeurs très élevées de dureté et du module élastique, et en particulier à basses températures, une fragilité bien plus grande que celle des métaux.

De point de vues de propriétés physiques, on notera que le carbure de silicium (SiC) présente une conductivité thermique relativement élevée comparée aux autres matériaux céramiques.

Les composites à base de SiC font l'objet de nombreux travaux dans le cadre du projet «Technologie fusion ». La difficulté de l'étude du comportement hors et sous irradiation provient du fait qu'il faut prendre en compte la contribution de chaque constituant: la matrice, les fibres et l'interface, cette dernière étant constituée d'une couche intermédiaire assurant la liaison entre la fibre et la matrice.

Les propriétés physiques et mécaniques de composites $\mathrm{SiC} / \mathrm{SiC}$ sont en général entachées d'une grande dispersion liée à la variabilité des fibres utilisées, aux méthodes d'infiltration de la matrice, au type de produit fabriqué. Lors de la dernière décennie, il y a eu des progrès significatif dans les procédés d'élaboration qui se sont traduits par une amélioration de la qualité des produits obtenus. Cependant, la capacité de production reste très limitée en termes de quantité et de forme des produits élaborés.

Les effets d'irradiation dans les céramiques sont plus complexes que dans les matériaux métalliques, du fait de la grande sensibilité de la liaison ionocovalente (excitation électronique, modification de composition chimique par transmutations...). Plusieurs phénomènes peuvent avoir lieu sous irradiation et 
sont fonctions de la température : gonflement, amorphisation, changement de phase, modification de la stoechiométrie, production de gaz (hydrogène et hélium), modifications de la mobilité ionique...

Les besoins en R\&D dans le domaine de composites céramiques portent sur :

- la mise au point et l'optimisation des procédés de fabrication afin d'obtenir différents types de produits avec microstructure et propriétés reproductibles;

- la compréhension de la relation microstructure et propriétés physiques/mécaniques, et en particulier, l'étude de la fragilité intrinsèque de ce type de matériau hors irradiation;

- modélisation du comportement mécanique à l'échelle adéquate ;

- le comportement sous irradiation dans une gamme large de températures d'irradiation (500-1200 ${ }^{\circ} \mathrm{C}$ ) ; l'évolution de propriétés physiques et mécaniques; la caractérisation de phénomènes induits par l'irradiation.

\subsubsection{Les matériaux pour le divertor}

Le développement de matériaux pour le divertor est fortement lié à l'évolution de la conception de celui-ci. La principale sollicitation subie par le composant est due au chargement thermique très important $\left(5-20 \mathrm{MW} / \mathrm{m}^{2}\right)$ et au dommage $\mathrm{d}^{\prime}$ irradiation ( $\left.20 \mathrm{dpa}\right)$.

Deux types de matériaux sont à considérer : les matériaux dits " fonctionnels » assurent la protection face au plasma et les matériaux de la structure elle-même qui auraient une interaction forte avec le caloporteur et qui doivent supporter des températures dans la gamme $600-1400^{\circ} \mathrm{C}$.

De par ses bonnes propriétés thermiques, sa résistance mécanique et sa tenue à l'érosion élevée, le tungstène et ses alliages sont considérés pour une application à long terme en tant que matériaux de protection et de structure. Les alliages base molybdène sont aussi retenus comme solution alternative.

Cependant, comme tous les matériaux métalliques réfractaires, la principale limitation réside dans la faible ductilité et ténacité du tungstène à des températures inférieures à $500^{\circ} \mathrm{C}$. Différentes approches sont étudiées afin de diminuer la fragilité intrinsèque à basses températures:

- la production du tungstène nanostructuré ; 
- le renforcement de la matrice par la dispersion de particules réfractaires;

- le contrôle et la réduction des teneurs en impuretés interstitielles.

Si les matériaux réfractaires sont bien produits à niveau industriel, beaucoup d'efforts sont encore nécessaires pour optimiser leurs performances.

D'autres matériaux tels que les alliages à base cuivre pourraient être utilisés pour les structures si le concept retenu pour le divertor le permet.

\subsection{Conclusion}

On peut signaler qu'un vaste programme de R\&D sur les aciers ferritiquesmartensitiques à activation réduite est en cours afin de valider et qualifier cette famille de matériaux pour les structures internes de réacteurs de fusion. Cette catégorie de matériaux est de loin la plus documentée et constitue une base de données appréciable pour de possibles applications à d'autres réacteurs nucléaires avancés.

Cependant, les différents concepts de structures internes d'un futur réacteur de fusion exigent le développement de solutions " matériaux 》 nouvelles qui puissent assurer de conditions de fonctionnement de plus en plus sévères. Notamment, la mise au point de matériaux pour fonctionner à haute température et supporter d'importants chargements neutroniques et électromagnétiques constitue le principal défi pour les spécialistes de la science des matériaux.

\section{3 | Les apports de la modélisation}

Récupérer de l'énergie d'un futur réacteur à fusion pose des problèmes inédits de matériaux.

En effet, du fait de l'énergie des neutrons produits par les réactions de fusion, la production d'hélium au sein des matériaux, par les réactions $(n, \alpha)$, est inévitable. Un ordre de grandeur souvent avancé pour un réacteur prototype est de $300 \mathrm{ppm}$ atomique d'He produit chaque année de fonctionnement dans les matériaux de structure les plus exposés. L'hélium étant insoluble dans les matériaux (autres que les gaz rares solides!), on pourrait s'attendre à une perte de cohésion de la matrice, si les atomes formés in situ restaient immobiles. 
Mais, comme c'est toujours le cas en métallurgie, les éléments insolubles précipitent. La cohésion et le comportement mécanique réels du matériau sont contrôlés par la microstructure de précipitation (ici, pour l'essentiel, I'hélium insoluble).

Voici donc un terrain de prédilection pour la modélisation multi-échelle des matériaux, domaine très en vogue, où les progrès les plus sérieux de la science moderne des matériaux côtoient les discours les plus démagogiques. On assiste à un développement foisonnant des techniques de modélisation, à toutes échelles :

- calculs ab initio des configurations atomiques et de leurs défauts, des vibrations, des collisions de métaux purs et dopés (entre 100 et 1000 atomes);

- simulations, par dynamique moléculaire, de processus rapides (jusqu'à la nanoseconde, sur quelques millions d'atomes) tels les cascades de déplacement résultant de l'irradiation, ou le glissement d'une dislocation ;

- simulation par diverses techniques de Monte-Carlo des cinétiques de vieillissement contrôlées par la diffusion : sur réseau cristallin en enchaînant les sauts de chaque espèce mobile, ou dans un continuum en enchaînant des évènements définis à une échelle spatiale supra-atomique dont les lois de probabilité sont calculées a priori (Monte-Carlo sur évènements, permettant de simuler des volumes de plusieurs microns au cube)...

- simulation par des modèles de cinétique chimique homogène ou hétérogène ("champs de phases ") de l'évolution des populations de défauts, de leurs amas, des précipités, des ségrégations...

- simulation de la dynamique du réseau de dislocations à trois dimensions (DDD : Discrete Dislocation Dynamics) et son couplage avec des calculs de contraintes par éléments finis...

- modélisation du comportement mécanique par les méthodes de la plasticité cristalline, permettant, entre autres, de tenir compte de la microstructure du matériau (forme et orientations respectives des grains), avec, dans certains cas, utilisation de la DDD pour modéliser la matrice d'écrovissage...

Ces techniques sont autant d'outils de compréhension du comportement des matériaux, en particulier sous irradiation, et dans certains cas, au prix d'un calibrage méthodique, peuvent fournir des méthodes d'extrapolation du comportement, à des conditions de sollicitation non accessibles à l'expérience. L'intérêt qu'elles présentent pour les matériaux pour la fusion est donc indéniable. 
Pour ce qui concerne le chargement en hélium des matériaux de structure, on sait que la morphologie de la précipitation (homogène ou hétérogène, fine ou grossière, avec ou sans contrainte associée aux précipités, etc.) est le résultat $d^{\prime}$ une compétition subtile entre trois facteurs :

- la sursaturation;

- la mobilité des espèces et leur mécanisme de diffusion (lacunaire, interstitiel direct ou indirect. . .);

- la nature des sites de germination (joints de grains, dislocations, amas divers de défauts ponctuels telles des microcavités. . .).

Lorsque ces trois facteurs sont connus, diverses techniques de modélisation permettent de prédire la morphologie de la précipitation, avec une bonne fiabilité. Ces techniques vont des diverses méthodes de Monte-Carlo cinétique à la théorie classique de la germination, en passant par les méthodes de dynamique d'amas. Ce ne sont pas encore des outils de type « boîte noire », mais un exercice coordonné au niveau national est en cours (2001-2006) pour en établir la fiabilité dans un contexte classique (CPR ${ }^{1}$ « Précipitation » entre CNRS, Arcelor, Pechiney et CEA sur la précipitation dans quelques alliages légers et dans des aciers au niobium). Les résultats montrent une bonne convergence des diverses techniques de modélisation.

Dans le cas des matériaux pour la fusion, la difficulté d'une prédiction provient de l'évolution dans le temps des trois facteurs ci-dessus et de leur interdépendance. En effet, la sursaturation s'établit progressivement au cours du temps, au gré de la compétition entre le terme source, que sont les réactions nucléaires, et la fuite que représente la précipitation elle-même. De plus, la nature et la densité des sites de germination évoluent du fait de la condensation des défauts ponctuels créés par l'irradiation; enfin, si la mobilité de He est affectée par les défauts ponctuels, elle dépendra elle aussi de l'évolution de cette condensation.

C'est pour ces raisons qu'on ne peut prétendre réaliser des expériences représentatives du comportement des matériaux dans ces futures machines, en absence d'un couplage étroit avec une modélisation réaliste ${ }^{2}$.

\footnotetext{
${ }^{1} \mathrm{CPR}$ : Contrat de programme de recherche.

${ }^{2}$ Jusqu'à un passé très récent, l'effort de développement de matériaux pour d'éventuels futurs réacteurs à fusion a ignoré cette nécessité. L'effort a été quasi uniquement expérimental et s'est fait dans la continuité des travaux sur la mise au point de matériaux pour les réacteurs rapides (à fission), matériaux dans lesquels, du fait du spectre d'énergie des neutrons, l'importance des réactions (n, a) peut être contrôlée en jouant sur la composition chimique (abandon des aciers austénitiques au profit des ferritiques).
} 


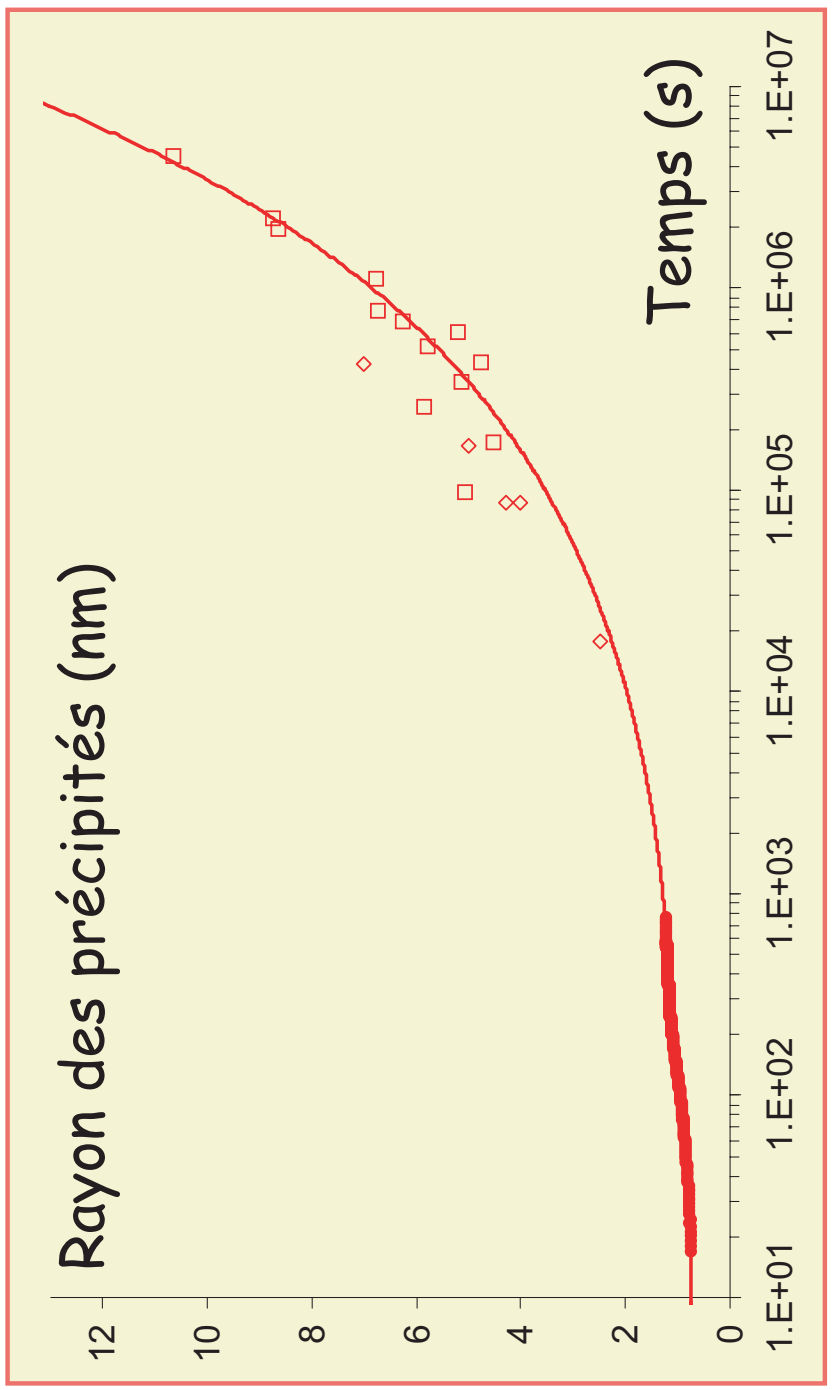

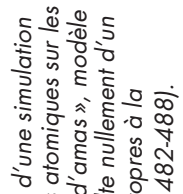

혹에

बै है क है के

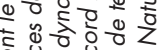

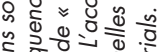

远造造

\&.

है

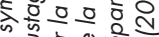

屯 흥 형

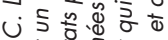

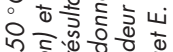

政

-

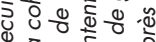

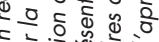

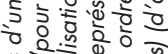

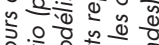

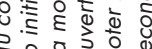

그응 으웡

पु

ㅎํㅇ율 है =

$\infty$ \& बें

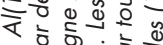

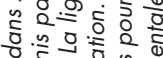

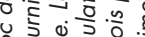

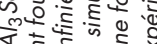

등ㄷㅇㅇ

童

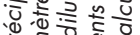

20. 등

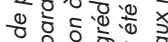

के क्र

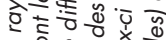

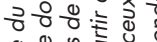

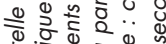

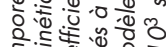

हن 0.0

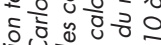

专讨

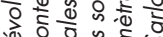
过定 운 है

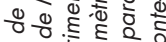

-

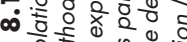

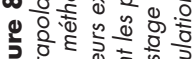

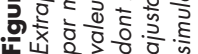


L'évaluation du terme source ne pose qu'un problème de fiabilité des calculs neutroniques et des valeurs de sections efficaces des réactions $(n, \alpha)$.

La fuite, quant à elle, est beaucoup plus délicate à estimer. La mobilité de $\mathrm{He}$ dans les matériaux est en effet mal connue et son mécanisme de diffusion encore controversé. Les calculs ab initio font en ce moment des progrès spectaculaires : les énergies de liaison entre He et divers défauts, et les barrières d'activation des divers mécanismes de diffusion deviennent calculables. Des modèles réalistes de défauts dans le fer (avec son magnétisme), en présence de carbone, sont maintenant accessibles: on s'approche de l'acier.
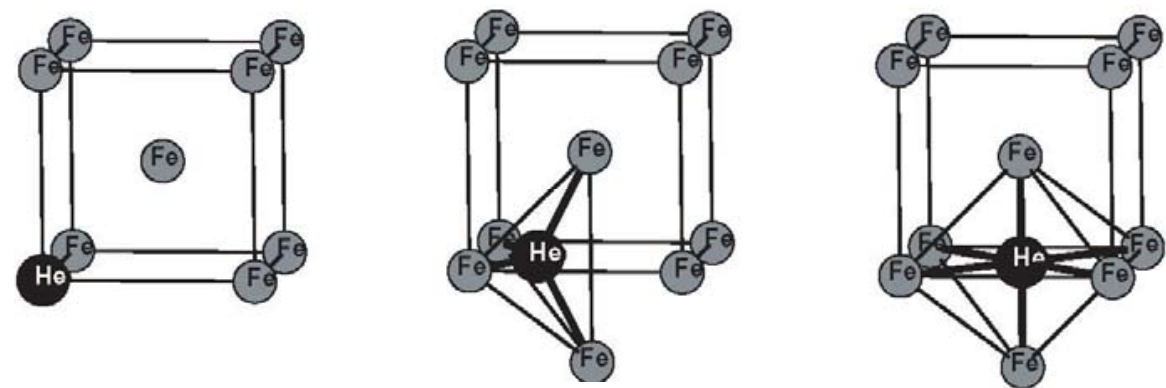

\section{Figure 8.2a}

Structure de diverses configurations de l'hélium, dans le fer $\alpha$, calculées par DFT-GGA avec magnétisme (code SIESTA) : les sphères noires symbolisent les atomes d'hélium. En présence de lacunes, la configuration la plus stable est substitutionnelle (figure de gauche). Les énergies calculées montrent que la configuration tétraédrique (au centre) est la plus stable.
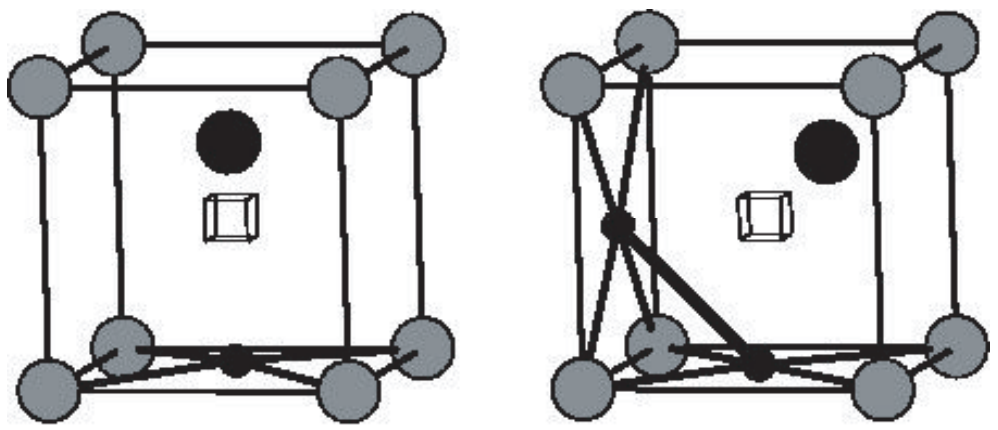

\section{Figure $\mathbf{8 . 2 b}$}

La présence de carbone (petite sphère sombre) affaiblit la liaison de l'hélium (grosse sphère sombre) à la lacune (au centre de la maille) : de 2,30 eV en absence de carbone, cette énergie s'abaisse à 2,09 et $0,94 \mathrm{eV}$ en présence respectivement d'un et deux atomes de carbone (d'après C.C. Fu et F. Willaime, CEA-Saclay). 
Le réalisme de ces calculs est attesté par la précision avec laquelle ils permettent de reproduire les stades de recuit de la résistivité du fer pur irradié à basse température, déterminés expérimentalement, il y a une vingtaine d'années (Chu Chun Fu et al., 2004). Les données précises sur le comportement de l'hélium sont malheureusement très rares, ce qui justifierait certainement la reprise de travaux expérimentaux sur matériaux bien caractérisés. Quelques équipes s'attaquent à la modélisation, particulièrement délicate, de l'alliage fer chrome, modèle des aciers ferritiques.

L'évolution de la densité des sites de germination de diverses natures lamas divers de défauts ponctuels tels des microcavités, dislocations, joints de grains. ..), peut être modélisée par plusieurs techniques : dynamique moléculaire, pour la création des défauts dans les cascades de déplacement qui résultent des collisions des particules incidentes avec les noyaux du matériau, diverses méthodes cinétiques pour la condensation des défauts en divers types $d^{\prime}$ amas et leur élimination sur les discontinuités du réseau cristallin (méthodes de Monte-Carlo cinétique sur réseau, sur objets ou sur évènements, dynamique d'amas, méthodes de champ moyen cinétique...). Ces méthodes, qui ont été décrites dans le rapport de l'Académie des sciences RST $N^{\circ} 5$ Matériaux du nucléaire (A. Zaoui, 2000), sont encore en développement. Plusieurs exercices coordonnés sont en cours en France et en Europe, pour en établir la cohérence dans quelques cas modèles : programme Sinergy de codéveloppement entre EDF, Framatome et le CEA, CPR Smirn entre CRNS, CEA et EDF, divers programmes européens (Euratom fission) dont le projet Perfect du $6^{\mathrm{e}}$ PCRD... Notons cependant que les niveaux d'irradiation envisagés pour la fusion sont importants : $25 \mathrm{dpa}^{3}$ par année de fonctionnement dans les matériaux de structure les plus exposés. La prise en compte, par ces modèles, de la production simultanée de défauts ponctuels et d'hélium reste un problème ouvert. Des innovations sont nécessaires pour permettre à ces méthodes d'aborder le cas d'alliages à nombreux constituants. En particulier, ces méthodes devraient, à terme, inclure le bilan de l'hydrogène créé par les réactions $(n, p)$. Un programme européen très modeste est en cours dans le cadre EFDA; I'UKAEA a lancé, au printemps 2004, un programme plus ambitieux dans ce domaine.

La microstructure étant prédite ou connue, que peut-on dire du comportement mécanique du matériau? C'est le domaine de la « métallurgie mécanique » et de la " mécanique de la rupture » où cohabitent des modèles simples " de métallurgistes », ayant fait leurs preuves, et des techniques de simulation lourde, en plein essor (dynamique des dislocations discrétisées, modèles de plasticité cristalline avec prise en compte des mécanismes de la plasticité, méthodes d'homogénéisation...). Ces développements ne sont pas spécifiques aux matériaux

\footnotetext{
${ }^{3} \mathrm{dpa}$ : déplacement par atome. C'est la fraction des atomes qui a été éjectées de leur site du fait d'une collision nucléaire. Dans les métaux, une forte proportion de ces atomes (50 à $90 \%$ suivant la structure cristalline) retrouve un site cristallin après quelques vibrations atomiques.
} 
pour le nucléaire, mais bénéficient du soutien d'une partie des programmes de recherche signalés ci-dessus. Les applications nucléaires réclament cependant des innovations (plasticité des alliages, prise en compte des interactions entre dislocations et amas de défauts créés par irradiation, montée des dislocations par absorption de défauts ponctuels ou amas mobiles. . .) et des calculs de grandeurs spécifiques au domaine (baisse de cohésion intergranulaire sous l'effet de ségrégations induites par l'irradiation. ..)

Ce qui précède concerne les matériaux de structure. La liste des défis posés à la modélisation n'est pas exhaustive : en particulier, pour le matériaux renforcés par des dispersions d'oxydes, se pose le problème de l'intégrité, sous irradiation, des particules dispersées : forme, taille, composition sont affectées par l'irradiation d'une manière qui est encore mal modélisée.

Pour les matériaux de protection se pose, en outre, le problème de l'érosion : érosion par cloquage (blistering) ou par pulvérisation. Le cloquage provient de la précipitation de l'hélium, près de la surface, et de l'éclatement des bulles sous l'effet de la pression interne. La modélisation de ce phénomène relève des outils évoqués ci-dessus. La pulvérisation, quant à elle, est plus difficile à modéliser, car toute une chimie sous rayonnement agit sur les rendements de pulvérisation; cette chimie met en jeu des transitions électroniques dont la modélisation se développe dans d'autres communautés (usinage par faisceaux d'ions, microélectronique...)

Enfin, un thème préoccupant, pour certains matériaux autres que les aciers, a jusqu'ici été laissé dans l'ombre : I'apparition de nouvelles espèces chimiques par réactions nucléaires (noyau mère dans les réactions $(n, \alpha)$ et $(n, p)$ ). Si de tels produits de transmutation ne présentent sans doute pas de danger dans les aciers, on est dans l'ignorance en ce qui concerne les matériaux céramiques. Une étude du FZK annonce la transmutation, en 100 dpa, d'environ $3 \%$ de $\mathrm{SiC}$ en $\mathrm{Al}$ et $\mathrm{Mg}$, éléments dont la solubilité dans (ou la réactivité avec) SiC est inconnue. Cette réflexion mérite d'être approfondie.

On le voit, la fusion stimule le domaine en pleine expansion qu'est la modélisation multi-échelle des matériaux. Les matériaux de structure mis en œuvre (aciers ferritiques) posent de nombreux problèmes difficiles : prise en compte du magnétisme dans les calculs ab initio ( $\mathrm{Fe}, \mathrm{Cr}, \ldots$. ), traitement efficace du carbone dans les modélisations de cinétiques, simulation du glissement des dislocations vis à des vitesses d'intérêt métallurgique, prise en compte, pour la plasticité, de la microstructure en lattes de la martensite, etc. Les quelques programmes coordonnés en cours, que nous avons cités ci-dessus, aident à focaliser les efforts avec un succès certain. 


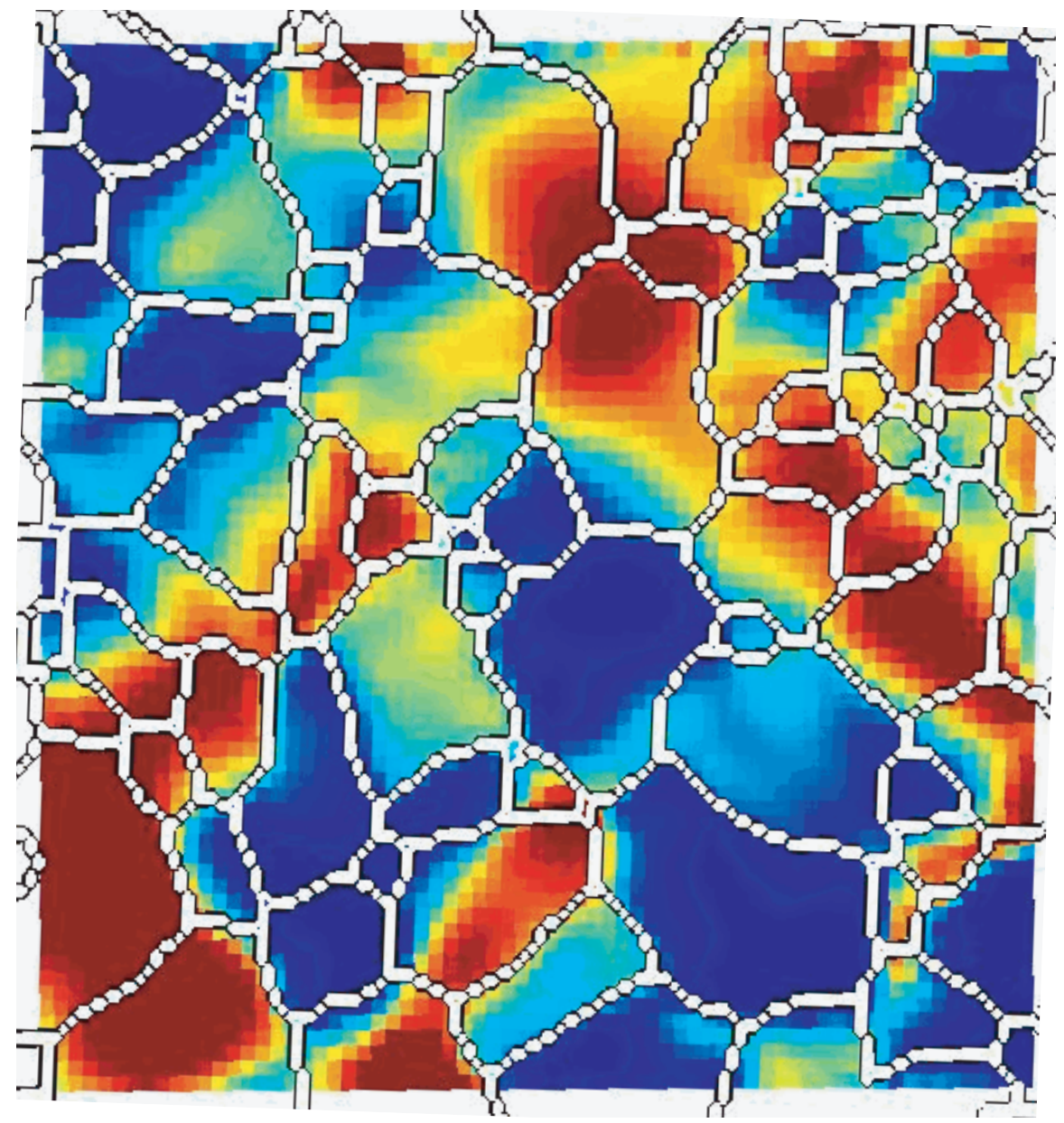

Figure $\mathbf{8 . 3}$

Carte de déformation équivalente (dans du zirconium) obtenue par simulation numérique pour un modèle élasto-viscoplastique, en plasticité cristalline : du bleu au rouge, la déformation passe de 1 à $7 \%$; les frontières blanches sont les joints de grains tels que révélés par la microscopie électronique à balayage; les orientations des grains contigus ont été déterminées par diffraction d'électrons rétrodiffusés (EBSD). Ces déformations peuvent être mesurées à l'aide d'une grille gravée à la surface de l'échantillon (d'après Dexet M. et al. (2004). CPR Smirn).

\section{4 | Les apports de l'expérimentation}

Comme on l'a vu, on ne peut prétendre réaliser des expériences représentatives du comportement des matériaux dans ces futures machines, en absence d'un couplage étroit avec une modélisation réaliste. Pour être représentatif, un résultat d'expérience devra être nécessairement extrapolé. 
Il existe plusieurs manières de charger un matériau en $\mathrm{He}$, avec des intensités d'irradiation simultanée diverses :

- chargement du matériau en tritium qui transmute en He par émission $\beta^{-}$;

- irradiation par des particules $\alpha$ dans un cyclotron à énergie variable, ou $\mathrm{d}^{\prime}$ autres machines;

- irradiation simultanée par plusieurs faisceaux d'ions (dont l'un de particules $\alpha$;

- irradiation neutronique d'alliages dopés en éléments à grande section efficace pour les réactions $(n, \alpha)$;

- irradiation dans des sources à spallation, dans des sources de neutrons de $14 \mathrm{MeV}$...

Pour les raisons évoquées au paragraphe précédent, chacune de ces méthodes conduira à une microstructure différente pour un chargement en hélium identique : en effet, le taux de production de l'hélium, le taux de production des défauts ponctuels (et leur mode de production en petites ou grosses cascades) ainsi que la température, contrôlent le chemin de développement de la microstructure.

Il faut donc se résoudre à considérer les moyens de chargement ci-dessus comme autant de tests des outils de modélisation, sans ignorer pour autant les phénomènes insoupçonnés qui peuvent se révéler au cours de tels traitements.

Dans ce contexte, l'irradiation simultanée de petits échantillons par plusieurs faisceaux de particules chargées et l'observation, à fine échelle (microscopie électronique post mortem ou in situ), de l'évolution de la microstructure résultante est sûrement le moyen le plus souple (et le moins onéreux) pour explorer rapidement une multitude de conditions de chargement et mettre à l'épreuve diverses méthodes de modélisation. Le projet Jannus du CEA est optimisé pour cet objectif4 ${ }^{4}$. Les études de propriétés mécaniques, quant à elles, doivent faire appel à des essais miniaturisés.

La réalisation d'expérimentations plus lourdes mais plus représentatives sera sans doute nécessaire. L'élaboration des critères de conception qui seront utilisés pour les réacteurs de fusion devra s'appuyer sur une base de connaissances validées où la qualification expérimentale complète semble incontournable, ne serait-ce que pour respecter les exigences qu'imposeront les autorités de sûreté.

\footnotetext{
${ }^{4}$ Trois faisceaux sont prévus dans le dispositif Jannus à Saclay : I'un pour implanter He, l'autre pour créer les défauts ponctuels, le troisième pour implanter l'hydrogène.
} 
À ce jour, en l'absence de source de neutrons de $14 \mathrm{MeV}$, la qualification expérimentale repose essentiellement sur l'utilisation des réacteurs à fission. $\mathrm{Ce}$ type de qualification a été mené pour les matériaux de structure $\mathrm{d}^{\prime}$ Iter (316L) et est en cours pour l'Eurofer qui sera utilisé pour les modules tests de couverture tritigène. Compatible avec lter et ses quelques dpa (et donc quelques appm de $\mathrm{He}$ ), ce type de qualification ne couvre pas les besoins du réacteur à fusion (de $150 \mathrm{dpa}$ et surtout une production d'He d'environ $10 \mathrm{appm} / \mathrm{dpa}$ ). La communauté internationale a donc proposé la construction d'une source de neutrons de $14 \mathrm{MeV}$ spécifique combinant d'une part un spectre neutronique "fusion", pour prendre en compte les effets de production de gaz qui $n^{\prime}$ apparaissent que pour des neutrons de haute énergie, et d'autre part une fluence importante qui permettent l'accès rapide à de fortes valeurs de dpa (100-200 dpa) sur un nombre d'échantillons représentatif.

Ce projet se nomme IFMIF (International Fusion Materials Irradiation Facility) et les travaux qui y sont liés sont réalisés sous l'auspice de l'IEA (International Energy Agency). Les partenaires majeurs sont l'Europe et le Japon mais le projet comprend aussi des contributions de la Suisse, des États-Unis, de la Russie et de la Chine.

IFMIF consiste en une source à neutrons basée sur les réactions ${ }^{7} \mathrm{Li}(\mathrm{d}, 2 \mathrm{n})^{7} \mathrm{Be}$, ${ }^{6} \mathrm{Li}(\mathrm{d}, \mathrm{n}){ }^{7} \mathrm{Be}$. L'accélérateur de $\mathrm{D}+$ est de type Linac (figure 8.4). Le courant nécessaire est généré par deux accélérateurs travaillant en parallèle. La cible Li convertissant le faisceau $D+$ en neutrons de haute énergie est constituée d'un jet de Li liquide circulant à la vitesse de 10-20 m/s. La chambre de test comporte 3 zones de fluence différente :

- zone 1 : zone de forte fluence (>20 dpa/an, $\mathrm{He} / \mathrm{dpa}=10 \mathrm{appmHe}$, volume $=0,5 \mathrm{~L}$ ) ;

- zone 2 : zone de fluence intermédiaire (1-20 dpa/an, volume = $6 \mathrm{~L}$ ) ;

- zone 3 : zone de faible fluence $(<1 \mathrm{dpa} / \mathrm{an}$, volume $=8 \mathrm{~L})$.

L'équipe projet a présenté son rapport de conception en janvier 2004. Le coût d'IFMIF est estimé à 88 millions de dollars en 2003 pour la phase d'ingénierie détaillée (4 ans) et 540 millions de dollars en 2003 pour la phase de construction qui se déroulerait en deux étapes:

- phase 1 ( 7 ans) : construction des bâtiments, de la boucle Li, d'un accélérateur en exploitation à $125 \mathrm{~mA}$, afin de tester les matériaux pour les modules de tests de couverture d'Iter, les corrélations des données fusionfission et des études génériques sur l'endommagement des matériaux; 


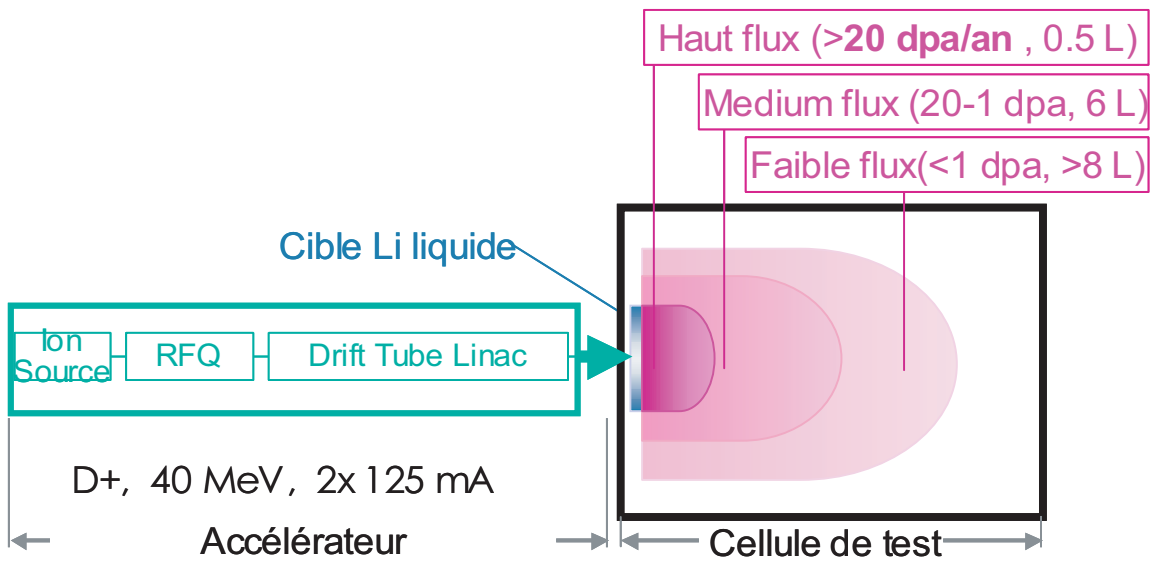

Figure 8.4

Schéma de principe du projet IFMIF.

- phase 2 ( 3 ans) : construction d'un second accélérateur de $125 \mathrm{~mA}$, permettant l'étude des performances des matériaux potentiellement utilisable dans un réacteur à fusion.

Évidement, ces expérimentations plus lourdes (irradiation dans des sources à spallation, les accélérateurs d'ions lourds à très fortes énergies, ou IFMIF, ...) ne trouveront pleinement leur sens que si les outils de modélisation sont prêts pour permettre d'extrapoler les résultats aux conditions attendues dans le futur réacteur. Les outils de modélisation rendront un outil comme IFMIF plus efficace en permettant de comprendre et de confirmer les résultats obtenus mais aussi en optimisant les conditions opératoires et en réduisant le nombre de campagnes expérimentales. La disponibilité d'outils de modélisation efficaces et des outils expérimentaux associés sont un gage de développement rapide de la fusion, qui devra le moment venu, être capable de démontrer aux autorités de sûreté que les matériaux utilisés ont été validés dans des conditions proches de leur utilisation réelle.

À titre d'exemple, la figure ci-dessous montre l'accroissement, avec la dose d'irradiation, du décalage de la température de transition ductile fragile de divers aciers. Comme on le voit, les irradiations à fort taux de chargement en hélium (symboles fermés), ne donnent pas de saturation à forte dose, dans le domaine de température étudié, contrairement aux irradiations par des neutrons " classiques». Mais la lourdeur du dispositif expérimental utilisé (source à neutrons de spallation) $n^{\prime}$ a pas permis de découpler les trois paramètres que sont la température, le flux d'irradiation et le taux de chargement d'hélium (ici très élevé : 60 ppm atomiques pour un déplacement par atome). Ceci illustre la 


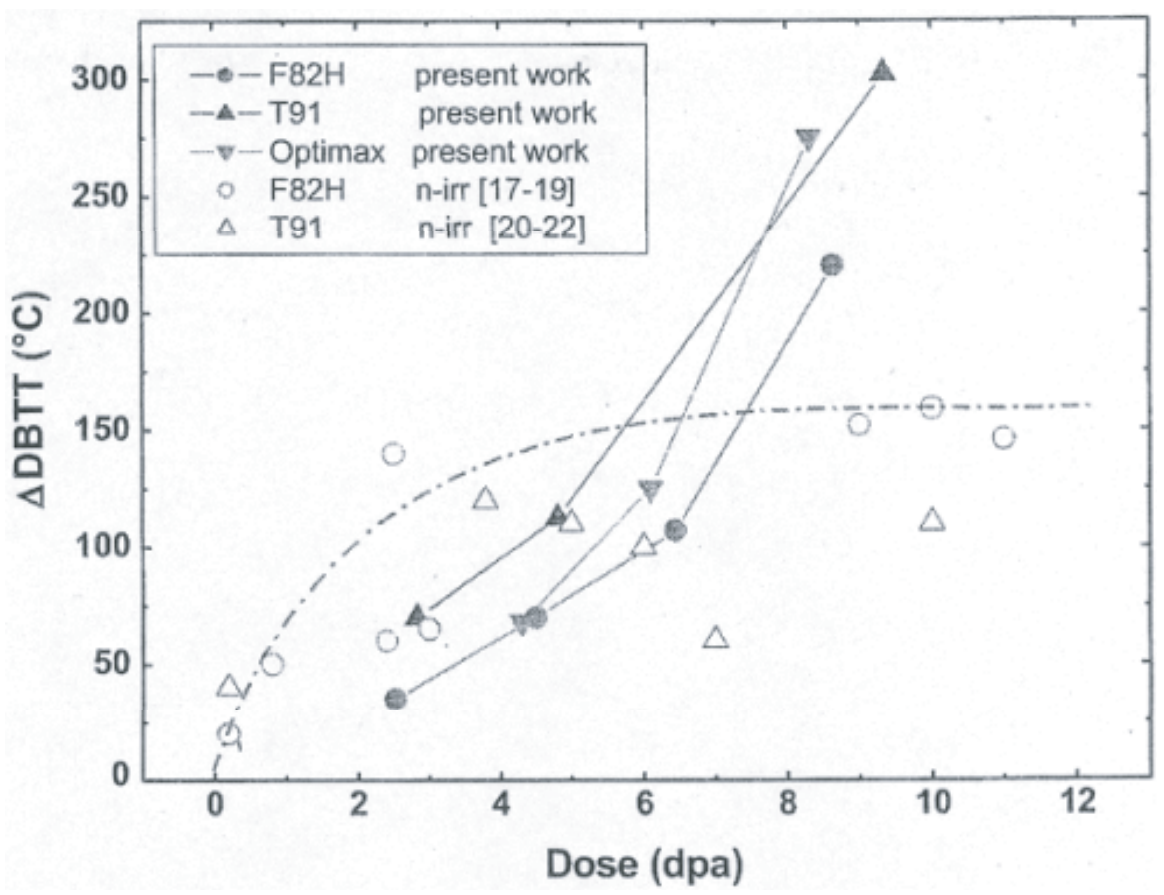

Figure 8.5

Décalages de la température de transition ductile/fragile pour divers aciers, en fonction de la dose d'irradiation (en déplacements par atome : dpa) dans deux types distincts d'irradiation : les irradiation neutroniques classiques (symboles ouverts) ou à fort taux de production d'hélium (60 appm He/dpa, symboles fermés) de la source à spallation SINQ au Paul Scherrer Institut. Suivant les essais, la température est comprise entre 80 et 275 Celsius (d'après Jia X. et Dai Y. (2003). J. Nucl. Mater. 323 : 360 ).

nécessité de disposer d'outils d'extrapolation calibrés sur des expériences couvrant de larges gammes de paramètres.

\section{5 | Les techniques d'assemblage}

L'assemblage interviendra à plusieurs niveaux dans la fabrication des structures modulaires entourant le plasma. Eu égard à leur complexité, les modules seront composés de différentes parties assemblées. De plus, différents matériaux seront très probablement nécessaires pour les modules (soit typiquement: un matériau de structure, éventuellement un matériau de puits thermique et un matériau résistant à l'érosion par le plasma). En outre, les modules seront assemblés à une structure arrière et alimentés en fluides par des tuyauteries. $\mathrm{Si}$ tous les assemblages devront respecter un cahier des charges strict, les cas les plus difficiles se trouvent dans les modules de par la sévérité des sollicitations 
thermomécaniques de ces composants, la complexité de leur géométrie, les problèmes de compatibilité métallurgique et mécanique des matériaux entre eux et les incertitudes sur le vieillissement des jonctions sous irradiation. Des techniques $\mathrm{d}^{\prime}$ assemblage variées seront utilisées dont le soudage, le soudage par diffusion et éventuellement le brasage. Le choix de la technique à retenir pour un besoin donné dépendra notamment de la nature des matériaux à assembler, de la géométrie, des critères $d^{\prime}$ inspection et de « réparabilité » des défauts ainsi que du degré d'automatisation requis.

\subsection{Les procédés d'assemblage par fusion}

Le matériau de structure d'Iter, l'acier austénitique $316 \mathrm{LN}-\mathrm{IG}$, possède une bonne soudabilité métallurgique. Une application majeure du soudage par fusion pour lter est l'assemblage des secteurs de la chambre en vide. Étant donné les épaisseurs concernées $(60 \mathrm{~mm})$, la technique de référence est le soudage TIG en chanfrein étroit. Ce procédé est qualifié et maîtrisé mais souffre d'une productivité faible. Des alternatives sont aujourd'hui en cours de développement: soudage par laser avec double source ou soudage hybride arc et laser. Les problèmes à résoudre sont les problèmes classiques de soudage : maîtrise des défauts, définitions des paramètres, gestion des déformations et définition des moyens de contrôle ultrasonore qui, dans le cas de ce matériau, posent un problème spécifique d'interférences ultrasons/joints de grains.

Une autre application importante concerne les connections tubulaires des composants internes de la chambre à vide. Là encore, le procédé TIG est une référence, mais des développements sur le soudage laser sont en cours. Étant donné les problèmes d'accessibilité (à travers les ports de la chambre à vide), on cherche à réaliser avec le même outil robotisé la découpe et le soudage, ceci dans l'optique de la réparation ou du remplacement de composants défectueux.

Le soudage par fusion est assez peu utilisé pour la fabrication des composants internes. On peut citer le soudage par faisceau d'électrons du Cu Cr Zr soit avec lui-même, soit avec l'acier 316LN par l'intermédiaire de nickel pur (composants du divertor).

En ce qui concerne les couvertures tritigènes en acier ferrito-martensitique, les développements sont moins avancés. La soudabilité métallurgique de ces aciers est plus délicate : présence de défauts lors du soudage en forte épaisseur, difficultés liées à la microstructure et à la nécessité de pratiquer après soudage un traitement thermique de détensionnement (jusqu'à présent insuffisamment efficace). Cela rend d'autant plus critique la nécessité de pouvoir contrôler les soudures réalisées, ce qui n'est pas systématiquement acquis, étant donné le dessin 
complexe des couvertures. De nombreuses incertitudes subsistent aujourd'hui sur la faisabilité du soudage en forte épaisseur, du détensionnement localisé (pour les connections tubulaires) en milieu irradié et de l'obtention de soudures plus résistantes à l'irradiation (présentant notamment une température de transition ductile-fragile suffisamment basse). Le soudage des aciers renforcés par dispersion d'oxydes (ODS) est un autre défi, cette classe de matériaux étant réputée non soudable par fusion.

De façon plus générale, la maîtrise des déformations demande encore des efforts de développement (soudage à l'arc en chanfrein étroit, soudage multi sources, ajout d'un refroidissement actif, ...) de même que le contrôle en ligne (positionnement de torche, profondeur de pénétration).

\subsection{Les procédés d'assemblage sans fusion}

Le soudage par fusion est peu adapté à la réalisation d'assemblages très hétérogènes, surtout quand les surfaces à assembler ne sont pas planes. II est fait appel à d'autres techniques ne mettant pas en œuvre la fusion du matériau de base : il s'agit du soudage par diffusion (à l'état solide) et du brasage.

Le soudage par diffusion est mis en œuvre en appliquant, à chaud, une force aux surfaces à assembler. Cette force est générée par une pression isostatique de gaz - compression isostatique à chaud - $(\mathrm{ClC})$. Les jonctions obtenues sont particulièrement résistantes. On notera que cette technique permet aussi la compaction de poudres et que la complexité des pièces qu'elle permet d'obtenir fait qu'elle relève plutôt $d^{\prime}$ 'une technique de mise en forme que d'assemblage uniquement. À partir de pièces élémentaires simples (plaques usinées, tubes cintrés ou non), on reconstitue un composant présentant des circuits de refroidissement interne qu'aucune autre technique ne permet d'obtenir. La CIC est une technique de référence pour la fabrication des panneaux de la couverture d'Iter composés $d^{\prime}$ acier austénitique, $d^{\prime}$ un alliage de cuivre et de béryllium. Le soudage diffusion est aussi une des techniques utilisées pour des composants à haut flux du divertor à base de tungstène et de cuivre.

Le brasage est utilisé pour assembler le matériau face au plasma (essentiellement le béryllium et les composites carbone-carbone) sur le puits thermique en alliage de cuivre. Des procédés de brasage spécifique ont été mis au point pour l'obtention de joints résistants. Pour les matériaux composites carbone-carbone, on utilise une préparation de surface consistant en une gravure par laser suivie d'une infiltration de cuivre. L'ancrage ainsi obtenu améliore les propriétés mécaniques et facilite le transfert thermique. Pour le béryllium, qui a tendance à former des intermétalliques fragiles avec le cuivre, on utilise le brasage rapide par induction à l'aide de brasures amorphes. Cela diminue la fragilité des jonctions. 
Les exemples ci-dessus concernent lter. Dans le cas des couvertures tritigènes, la complexité géométrique est plus grande, en particulier dans le cas où I'hélium est choisi comme fluide caloporteur (petitesse des canaux). Le soudage par diffusion reste une technique de première importance mais il faudra certainement la coupler avec le soudage par fusion : il en découle la nécessité d'étudier l'influence $d$ 'une technique sur l'autre et, finalement, $d$ 'avoir une approche globale de la gamme de fabrication de l'objet. L'application du soudage par diffusion aux ODS est une solution prometteuse pour palier les difficultés rencontrées avec les techniques de soudage par fusion. II est aujourd'hui envisageable de « plaquer » une couche d'ODS sur les parties les plus exposées au fluage des modules en acier ferrito-martensitique, notamment la première paroi.

Le brasage n'a aujourd'hui pas d'application au cas des couvertures tritigènes car la composition des brasures (souvent à base de nickel, de cuivre, $d^{\prime}$ 'argent, de bore) les rend incompatibles avec l'environnement neutronique. Le développement de nuances de brasures à faible activation sera peut-être rendu nécessaire par l'évolution des concepts. On pense notamment à l'assemblage des composites à base de carbure de silicium, car pour ces matériaux il existe peu d'alternatives au brasage.

De façon générale, la mise au point de l'assemblage requiert certes des études de base en laboratoire (définition des paramètres opératoires, détermination des propriétés mécaniques. ..) mais aussi des tests de qualification sur des maquettes représentatives, notamment des tests thermomécaniques simulant les conditions d'utilisation des composants dans les réacteurs, sans oublier qu'en situation réelle les composants seront soumis à l'irradiation. Le calcul numérique intervient à plusieurs niveaux, de la simulation du procédé d'assemblage à la modélisation des tests de maquettes en passant par la modélisation du comportement mécanique des jonctions.

\section{Références bibliographiques}

Chu-Chun Fu, Dalla Torre J., Willaime F., Bocquet J.L., Barbu A. (2003). Multiscale modelling of defect kinetics in irradiated iron. Nature Materials, 4 : 68-74.

Académie des sciences (2000). RST № 5 Matériaux du nucléaire. Animateur : André Zaoui. Éditions Tec \& Doc, Paris, pp. 37-92.

M. Dexet et al. (2004). Compte rendu d'avancement au CPR Smirn.

Jia X., Dai Y. (2003). J. Nucl. Mater., 323 : 360. 
